\title{
FOAM ACCUMULATORS: PACKAGING AND WEIGHT REDUCTION FOR MOBILE APPLICATIONS
}

\author{
Manuel Rexer ${ }^{1}$, Peter Kloft ${ }^{2}$, Frank Bauer ${ }^{2}$, Jakob Hartig ${ }^{1}$, Peter F. Pelz ${ }^{1 *}$ \\ ${ }^{1}$ Institut für Fluidsystemtechnik, Technische Universität Darmstadt, Otto-Berndt-Straße 2, 64287 Darmstadt \\ ${ }^{2}$ HYDAC TECHNOLOGY GmbH, Justus-von-Liebig Straße, Werk 2, 66280 Sulzbach/Saar \\ * Corresponding author: Tel.: +496151 1627100; E-mail: peter.pelz@,fst.tu-darmstadt.de
}

\begin{abstract}
Standardized parts like hydraulic accumulators are used in nearly every hydraulic system, in many cases even several. Therefore, even small changes in size and weight of accumulators can save considerable material costs. In mobile applications, hydraulic accumulators are used among others in hydro-pneumatic suspension systems. There is a strong focus on miniaturization and weight reduction, as the components always have to be transported with the vehicle. Energy density and energy content of conventional hydraulic accumulators cannot be maximized at the same time. This limitation can be overcome by adding a heat capacity with large surface into the gas volume of the accumulator. The heat capacity enlarges the isothermal frequency range and therefore enlarges the energy density of the accumulator at the given frequency and the given size.

In this paper an experimental comparison of conventional hydraulic accumulators and accumulators with foam inserts shows, that at a specific frequency band, the stiffness of foam filled accumulators is significantly lower than of conventional accumulators. The energy density is about $11 \%$ higher than in conventional accumulators. Consequently, a space reduction of about $18 \%$ is possible.
\end{abstract}

Keywords: foam, hydraulic accumulator, heat sink, hydropneumatic spring,

\section{INTRODUCTION}

In mobile hydraulics there is a trend to miniaturization and integration of functions to reduce package and external complexity. But not all vehicles are built in sufficient quantities to justify custom built highly integrated hydraulic components. Therefore, standardized parts like hydraulic accumulators should not be overlooked in miniaturization efforts.

Hydraulic accumulators are used to store energy in nearly every fluid system, e.g. for dynamic energy demand. In mobile applications hydraulic accumulators are used among others in hydro-pneumatic suspension systems (see Fig. 1) to store potential energy. For these applications space and weight reduction by smaller components is inevitable due to material costs and energy demand.

In this paper hydraulic accumulators with foam in the compressible gas volume are investigated for their space saving potential in mobile applications. Since hydro-pneumatic suspension systems are vibrating systems the stiffness transfer behavior over frequency has to be considered.

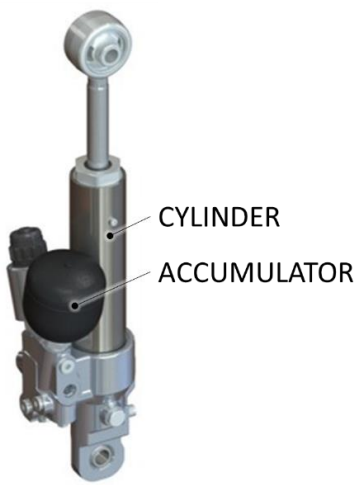

Figure 1: Example for a hydro-pneumatic suspension. 


\section{METHOD}

\subsection{Accumulators in hydropneumatic suspension systems}

Suspension systems such as those used in vehicles are meant to "carry loads", "store energy" and "damp". Hydro-pneumatic suspension struts consist of at least one hydraulic cylinder and one hydraulic accumulator connected by a throttle. Fig. 2 shows the basic structure of the hydro-pneumatic strut with level control.

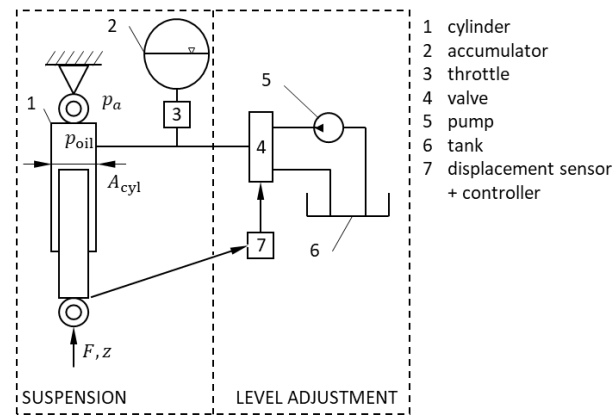

Figure 2: Principle of a hydro-pneumatic suspension. (in accordance with [1])

The hydraulic accumulator thereby fulfills the function of carrying load and storing energy. In the following damping is left out of the equation but can be taken into account using simple models. The force of the hydro-pneumatic strut can be expressed as

$F=A_{\text {cyl }}\left(p_{\text {oil }}-p_{\text {a }}\right)$.

Since the area of the cylinder $A_{\text {cyl }}$ and ambient pressure $p_{\mathrm{a}}$ are constant and the throttle is neglected the force is only determined by the pressure in the oil $p_{\text {oil }}$. A stiffness $K$ for the hydraulic accumulator can be specified independently of the cylinder surface $[1,2]$

$K=\left.\frac{1}{A_{\text {cyl }}^{2}} \frac{d F}{d z}\right|_{z=0}=\left.\frac{d p}{d V}\right|_{z=0}=n \frac{p_{1}^{2}}{p_{0} V_{0}}$.

The stiffness thus depends on the load pressure $p_{1}$, the precharge pressure $p_{0}$ and the initial gas volume $V_{0}$. For comparison purposes the stiffness is de-dimensioned with

$K^{+}=K \frac{p_{0} V_{0}}{p_{1}^{2}}$.

Therefore, fluctuations in the precharge- and load pressure in the measurement vanish. A polytropic state change with the polytropic exponent $n$ is assumed

$\left(\frac{p_{1}}{p_{2}}\right)=\left(\frac{T_{1}}{T_{2}}\right)^{\frac{n}{n-1}}=\left(\frac{V_{2}}{V_{1}}\right)^{n}$.

The polytropic exponent can be specified for the limiting cases of isothermal and adiabatic state changes as

$n=\left\{\begin{array}{l}1: f \rightarrow 0 \\ \gamma: f \rightarrow \infty\end{array}\right.$

For nitrogen the isentropic exponent $\gamma$ is about 1.4.

The load pressure $p_{1}$ and the precharge pressure $p_{0}$ of the accumulator are given by the design and can only be changed to a limited extent [1]. In order to change size or weight while keeping stiffness constant, the polytropic exponent $\mathrm{n}$ must be adapted.

The transition between isothermal and adiabatic state change is characterized by a thermal relaxation time, i.e. the ratio of thermal conductivity to thermal capacity. The heat capacity $V_{0} c_{\mathrm{v}} \varrho_{0}$ is that of the gas in the accumulator and the conductivity $k A$ is given by the transition of heat through the wall [3]. The relaxation time yields

$\tau=\frac{V_{0} c_{\mathrm{V}} \varrho_{0}}{k A}$.

The transition frequency $f_{\tau}$ between isothermal and adiabatic behavior of the accumulator can be read in the frequency response of the dimensionless stiffness at the point of the largest slope (see Fig. 3).

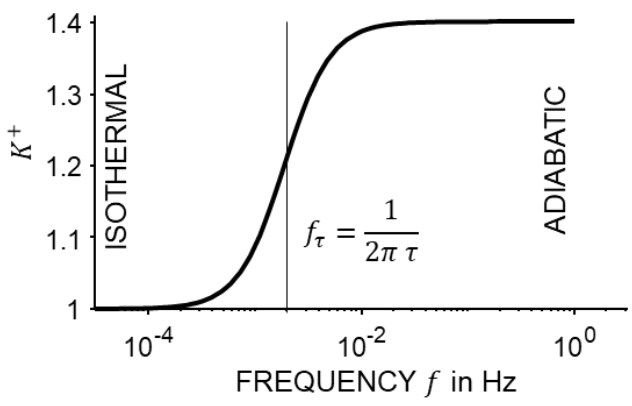

Figure 3: Frequency response of dimensionless stiffness.

As previous studies show foam is assumed to change heat transfer properties of accumulators by being a large heat capacity with large surface 
area and thus moving the transition from isothermal to adiabatic behavior [4]. Investigations also show that foam can increase the efficiency of accumulators [5].

\subsection{Hydraulic accumulators with foam inserts}

Foams are porous media that can be described with two characteristic quantities, porosity and specific surface area. A detailed picture of the polyurethane foam structure filled in the gas volume of the accumulator can be seen in Fig. 4. The cells of the foam are open and the pores have a diameter in the order of $0.1 \mathrm{~mm}$.

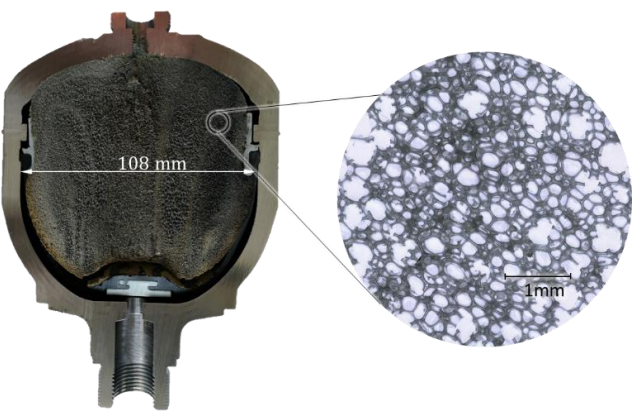

Figure 4: Section through a foamed hydraulic accumulator and detailed structure of the filled foam.

The porosity $\varphi$ indicates a volume ratio between the gas $V_{\mathrm{s}}$ and the total volume of the accumulator $V_{\mathrm{s}}+V_{\mathrm{g}}$ and is defined as

$\varphi:=\frac{V_{\mathrm{g}}}{V_{\mathrm{g}}+V_{\mathrm{S}}}$.

Since the stiffness of the accumulator depends on the gas volume (cf. Eq (2)), the porosity can be used to characterize the influence of the foam.

The specific surface area is a measure of the size of the individual foam cells. It indicates the ratio between surface area $A_{\mathrm{s}}$ and volume of the solid $V_{\mathrm{S}}$

$S_{\mathrm{S}}:=\frac{A_{\mathrm{S}}}{V_{\mathrm{S}}}$.

For a sphere with the diameter $d$ the specific surface area is given by $s_{S}=6 / d$.

The larger the specific surface of the foam, i.e. the smaller the pores, the faster a heat exchange between gas and foam takes place. The thermal relaxation time can therefore be shortened.

\subsection{Test rig}

In order to investigate the dynamic properties of hydraulic accumulators a test rig was developed. The principle of the test rig is shown in Fig. 5 . The basic design is similar to a hydro-pneumatic suspension strut.

A single-acting hydraulic cylinder is displaced in a path-controlled manner. The oil is moved into the accumulator to be tested. The resulting pressures in oil and gas are measured. The stiffness of the accumulator can thus be determined during harmonious excitation.

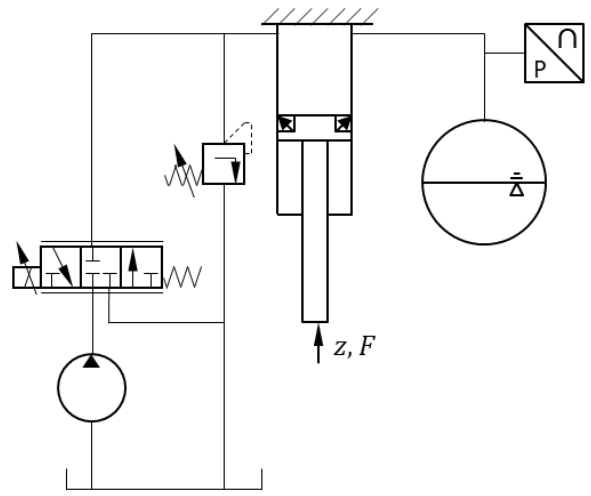

Figure 5: Schematic diagram of the develop test rig.

In addition, a level control system is integrated in the test rig. With a pump and a 3-3-way valve, the working point of the accumulator can be adjusted. In order not to overload the test bench, safety devices are integrated.

The real structure can be seen in Fig. 6. The displacement of the cylinder is controlled by a single-axis servo-hydraulic testing.

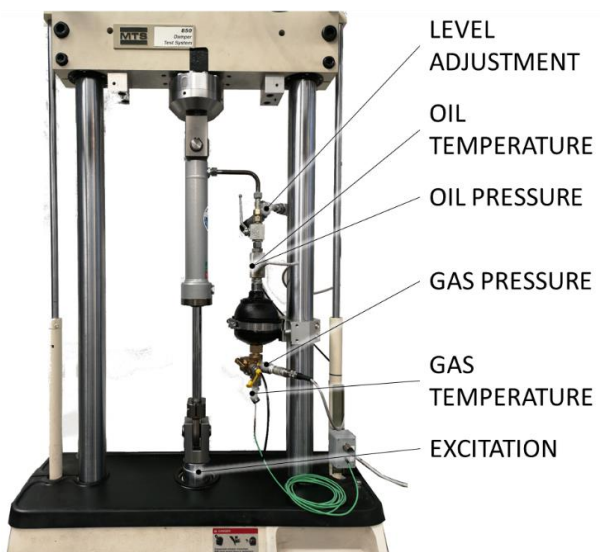

Figure 6: Test rig with level adjustment. 


\section{Test rig properties}

The features of the test bench are shown in Table 1. The values given are the maximum values.

Table 1: Properties of the designed test rig.

\begin{tabular}{ll}
\hline Test rig parameter & Value \\
\hline pressure & $250 \mathrm{bar}(\mathrm{abs})$ \\
oil flow rate & $1 \mathrm{1} / \mathrm{s}$ \\
exchange oil volume & $\pm 300 \mathrm{ml}$ \\
\hline
\end{tabular}

\section{Measured values}

The quantities listed in Table $\mathbf{2}$ are measured as shown in Fig. 6.

Table 2: measured values

\begin{tabular}{lll}
\hline symbol & measured value & unit \\
\hline$p_{\text {oil }}$ & oil pressure & bar $(\mathrm{abs})$ \\
$p_{\text {gas }}$ & gas pressure & bar $(\mathrm{abs})$ \\
$T_{\text {oil }}$ & oil temperature & ${ }^{\circ} \mathrm{C}$ \\
$T_{\text {gas }}$ & gas temperature & ${ }^{\circ} \mathrm{C}$ \\
$T_{\mathrm{a}}$ & ambient temperature & ${ }^{\circ} \mathrm{C}$ \\
$\Delta \mathrm{V}$ & excitation & $\mathrm{ml}$ \\
\hline
\end{tabular}

\subsection{Evaluation Method}

The stiffness of an accumulator is defined as the gradient $\mathrm{dp} / \mathrm{dV}$ of the force-displacement characteristic. Due to the non-linear behavior of an accumulator the stiffness is not constant over the amplitude. Nevertheless, a stiffness characterizing the accumulator should be specified for comparison purposes.

This is possible by defining the stiffness as the diagonal slope of an enclosing rectangle

$K^{+}:=\frac{p_{\max }-p_{\min }}{V_{\max }-V_{\min }}$

As long as the accumulator behaves like a linear system the hysteresis curves are elliptical. In this case the slope around the zero position corresponds to the slope of the diagonal (c.f. Fig. 7). [6]

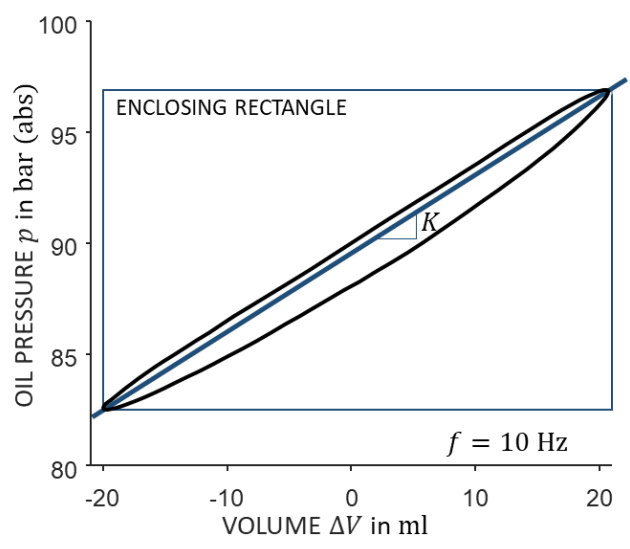

Figure 7: Elliptical hysteresis at $10 \mathrm{~Hz}$ with stiffness $K$.

As the amplitudes increase the accumulator no longer reacts harmoniously. As a result the hysteresis curves lose their elliptic shape and show a progressive course. In order to be able to calculate a characteristic stiffness in this case, evaluation windows are useful. Details can be found in [6]. For this paper only nearly elliptical hysteresis curves have been considered and therefore the enclosing rectangle method was chosen.

To determine the stiffness the accumulators have been excited with several oscillation periods and only the last full period was considered in the evaluation. This assures that the transient response of the accumulator has decayed.

\section{RESULTS}

The dimensionless stiffness determined at different frequencies as shown in chapter 2.4 can be displayed in a stiffness-frequency diagram.

In Fig. 8 the dimensionless stiffness (eq. 3) of conventional and foamed accumulators is shown as a function of frequency. In the isothermal frequency range foamed accumulators have a larger stiffness than conventional accumulators. In the adiabatic frequency range the stiffness of the foam accumulators is lower than the stiffness of the conventional hydraulic accumulator for all load pressures. For the frequencies between 1 and $10 \mathrm{~Hz}$ an increase in stiffness can be seen in both accumulators. This is due to the different measurements being taken one after another which leads to a temperature increase. 


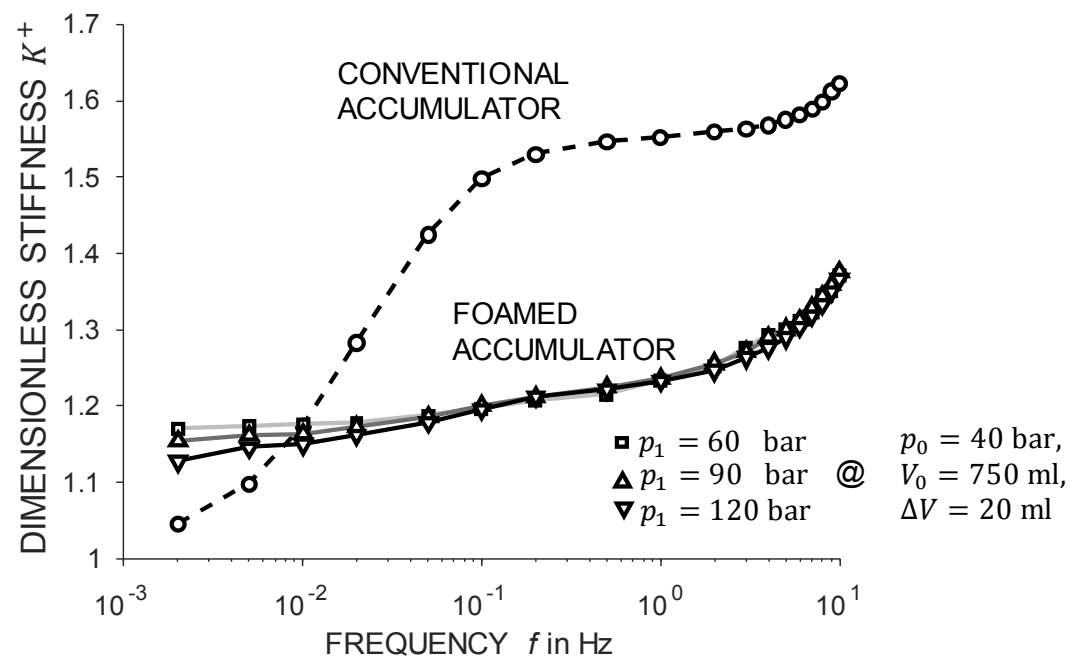

The foam in the accumulator has three effects on its function: (i) reduction of gas volume, (ii) foam mechanics and (iii) added heat capacity.

(i) The porosity of the foam is about $94 \%$ at atmospheric pressure. Thus the volume of the foamed accumulator is $6 \%$ lower than in the conventional accumulator. This leads to a stiffer accumulator recognizable in the isothermal frequency range.

(ii) The foam mechanics add to the stiffness of the accumulator. With a foam accumulator connected to the ambient air the stiffness was measured to be in the realm of $10^{-2} \mathrm{bar} / \mathrm{ml}$. This however is one magnitude lower than the stiffness of the gas.

(iii) The added heat capacity is a heat sink with a large surface. In the isothermal range this heat capacity has no effect since the accumulator is isothermal. In the adiabatic range the temperature rise with compression is lower with the foam since heat can be stored in the foam.

It can be concluded that there is a lower stiffness in foamed hydraulic accumulators even though the foam reduces the gas volume and mechanically adds to the stiffness. The reason for this is the heat capacity. However, impacts of the foam on the mechanics of the moving membrane have not been considered.
With these results an approximate polytropic exponent (c.f. eq. 3) for foamed accumulators can be calculated to be $n=1.15$. This exponent can be used for design calculations for hydropneumatic suspensions systems with foamed accumulators.

\subsection{Energy density}

In order to be able to classify the accumulators among themselves and in comparison to alternative energy storage options, the energy density is suitable as a benchmark. The energy density $w$ is the ratio of the storable energy $W_{\text {acc }}$ to the volume of the accumulator $V_{\text {acc }}$

$w=\frac{W_{\text {acc }}}{V_{\text {acc }}}$ [7]

The specific energy $\varrho_{\mathrm{W}}$ is the storable energy $W_{\text {acc }}$ in relation to the mass of the accumulator $m_{\text {acc }}$

$\varrho_{\mathrm{W}}=\frac{W_{\mathrm{acc}}}{m_{\mathrm{acc}}} .[7]$

To estimate these quantities a spherical accumulator with the specifications in Table 3 is assumed. The mass of the accumulator was determined from the minimum required wall thickness of a steel sphere that can resist the required pressure according to the Barlow's formula. 
Table 3: specifications of the accumulator.

\begin{tabular}{lll}
\hline symbol & parameter & value \\
\hline$m_{\text {acc }}$ & mass & $76.4 \mathrm{~g}$ \\
$d_{\text {acc }}$ & diameter & $124 \mathrm{~mm}$ \\
$V_{\text {acc }}$ & volume & 11 \\
$p_{\max }$ & maximum pressure & $300 \mathrm{bar}(\mathrm{abs})$ \\
\hline
\end{tabular}

The energy stored in a hydraulic accumulator during a charging process from precharge pressure $p_{1}$ to pressure $p_{2}$ as can be seen in Fig. 9 is given by

$|W|=\int_{1}^{2} p \mathrm{~d} V$.

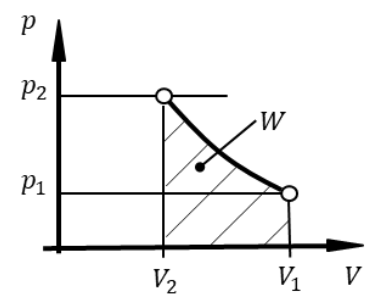

Figure 9: Stored energy of a hydraulic accumulator diagram when charging from $p_{1}$ to $p_{2}$.

For polytropic state changes (see equations (3) and (4)) with the polytropic exponent $n$, the maximum storable energy can be specified as

$W_{\text {poly }}=\frac{p_{1} V_{1}}{n-1}\left(1-\left(\frac{p_{1}}{p_{2}}\right)^{\frac{1-n}{n}}\right)$

Analogously for isothermal state changes this applies to the stored energy

$W_{\text {iso }}=p_{1} V_{1} \ln \left(\frac{p_{2}}{p_{1}}\right)$.

For a constant given volume $V_{1}=V_{\text {acc }}$ and pressure $p_{2}=p_{\max }$, there is an optimum precharge pressure $p_{1}$ at which the most energy can be stored in the accumulator. This optimum precharge pressure can be determined by deriving and setting to zero the energy in the accumulator as shown in [3] and was used to calculate the energy here.

In Fig. 10 the energy density is given as a function of the specific energy. The higher the values for energy density and specific energy, the more efficient the accumulator. A foam accumulator of the same size can therefore store $11 \%$ more energy than an adiabatic accumulator.

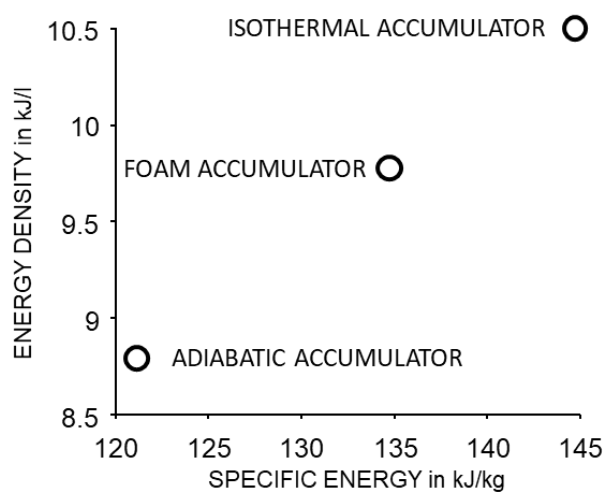

Figure 10: Energy density diagram for conventional and foam hydraulic accumulators.

In Fig. 10, the accumulators are each examined at their optimum frequency. For the application in the vertical dynamics of vehicles, frequencies in the range between $0.1 \mathrm{~Hz}$ and $25 \mathrm{~Hz}$ mainly occur [8]. A conventional accumulator therefore does not operate isothermal in this frequency range. In Fig. 11, the energy density is plotted over the frequency at which the accumulator can operate.

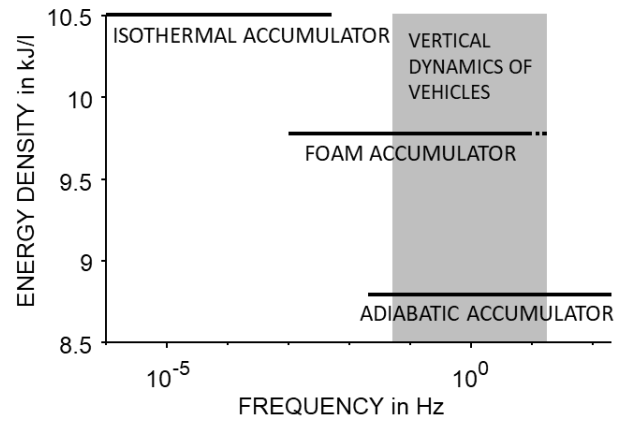

Figure 11: Energy density of foam and conventional accumulators at their operate frequencies.

It is shown that a foam accumulator is suitable for applications in vehicle suspensions and has a higher energy density than a conventional accumulator which operates adiabatically in this frequency range.

A one-mass oscillator is used to investigate the influence of the foam on a hydro-pneumatic suspension in a vehicle application. This simple vibrating system, is considered to be a model for a chassis.

According to equation (2) the volume of the accumulator can be calculated as a function of the natural frequency for foamed and conventional 


\section{ONE MASS OSCILATOR}
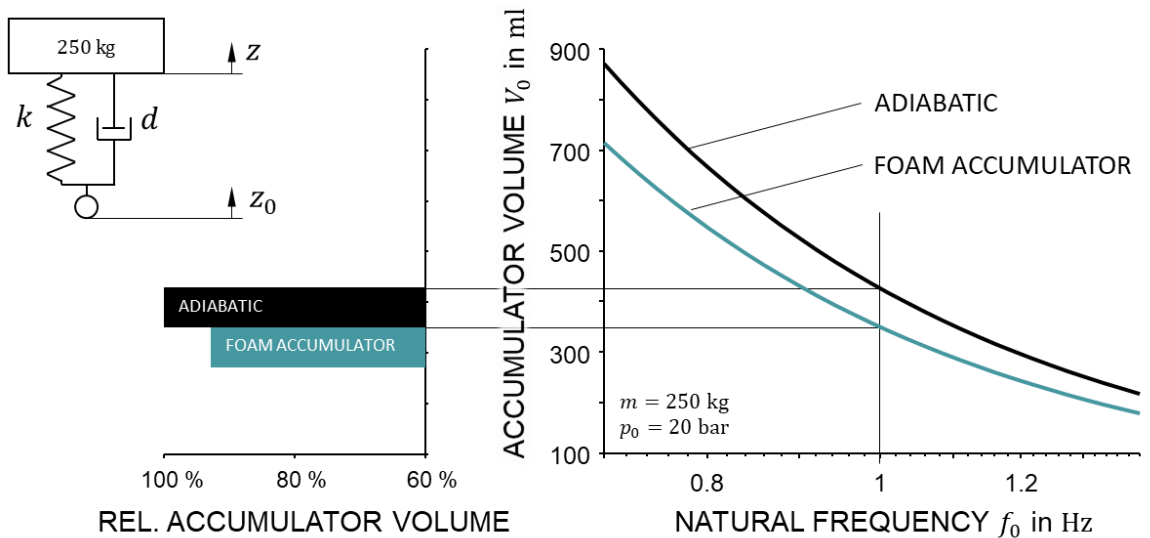

Figure 12: Accumulator volume of foam and conventional accumulators in one mass oscillators.

accumulators (cf. Fig. 12). The load pressure $p_{1}$ is calculated from the mass $m$ and the gravity $g$ to yield

$p_{1}=\frac{m g}{A_{\text {cyl }}}$.

At a natural frequency of $1 \mathrm{~Hz}$, which is typical for vehicles, $18 \%$ volume can be saved with a foam accumulator. A small accumulator not only has a smaller package, but also lower weight and lower material consumption.

\section{CONCLUSION}

It can be concluded that foamed hydraulic accumulators have a lower stiffness and consequently a higher energy density for vibrational applications. This is important for applications in hydro-pneumatic suspension systems. The reason for this behavior is the foam acting as a transient heat sink.

\section{ACKNOWLEDGMENTS}

Funded by the Deutsche

Forschungsgemeinschaft (DFG, German

Research Foundation) - Projektnummer 57157498 - SFB805.

\section{NOMENCLATURE}

A accumulator surface area

$A_{\text {cyl }} \quad$ area of the hydraulic cylinder

$A_{\mathrm{s}} \quad$ foam surface area

$\gamma \quad$ isentropic exponent $c_{\mathrm{v}} \quad$ isochoric Exponent of the gas

$d \quad$ diameter of a sphere

$d \quad$ damping constant

$d_{\text {acc }} \quad$ diameter of the accumulator

$f$ function

$f \quad$ frequency

$f_{0} \quad$ natural frequency

$g \quad$ gravity

$F \quad$ force of the hydro-pneumatic strut

$k \quad$ conductivity for transition through the wall

$K \quad$ stiffness

$K^{+} \quad$ dedimensioned stiffness

$m$ mass

$m_{\text {acc }}$ mass of the accumulator

$n \quad$ polytropic exponent

$p \quad$ pressure

$p_{\mathrm{a}} \quad$ ambient pressure

$p_{\max } \quad$ maximum pressure

$p_{\min } \quad$ minimum pressure

$p_{\text {gas }} \quad$ gas pressure

$p_{\text {oil }} \quad$ oil pressure

$p_{0} \quad$ precharge pressure

$p_{1} \quad$ load pressure

$p_{1} \quad$ inlet pressure of state change

$p_{2} \quad$ outlet pressure of state change

$\varphi$ porosity

$\varrho_{0} \quad$ density of the gas

$\varrho_{\mathrm{W}} \quad$ specific energy

$S_{\mathrm{s}} \quad$ specific surface area

$T_{\mathrm{a}} \quad$ ambient temperature

$T_{\text {gas }} \quad$ gas temperature

$T_{\text {oil }} \quad$ oil temperature

$T_{1} \quad$ inlet temperature of state change

$T_{2} \quad$ outlet temperature of state change 


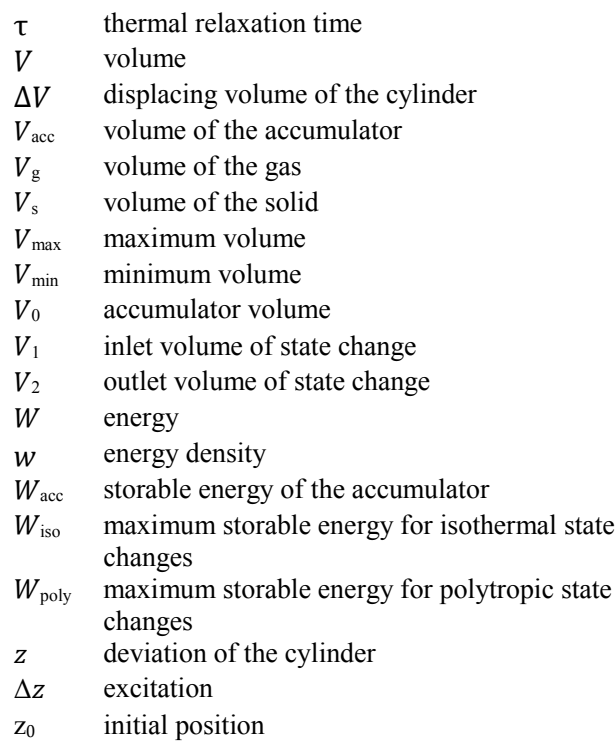

\section{REFERENCES}

[1] Bauer W (2008) Hydropneumatische Federungssysteme. VDI-Buch, Berlin, Heidelberg

[2] Pelz PF, Buttenbender J (2004) The dynamic stiffness of an air-spring International Conference on Noise and Vibration Engineering. Katholieke Universiteit Leuven - Departement Werktuigkunde, S 1727-1736
[3] Korkmaz F (1982) Hydrospeicher als Energiespeicher. Springer Berlin Heidelberg, Berlin, Heidelberg

[4] Stroganov A, Sheshin L (Hrsg) (2011) Improvement of Heat-Regenerative Hydraulic accumulators, Bd 11

[5] Geimer M, Synek P-M (2015) Hybride und energieeffiziente Antriebe für mobile Arbeitsmaschinen Arbeitsmaschinen. 5. Fachtagung, 25. Februar 2015, Karlsruhe. Karlsruher Schriftenreihe Fahrzeugsystemtechnik, Bd 30. KIT Scientific Publishing; Technische Informationsbibliothek u. Universitätsbibliothek, Hannover, Karlsruhe

[6] Puff M, Pelz PF (2009) Entwicklung einer Prüfspezifikation zur Charakterisierung von Luftfedern. FAT-Schriftenreihe. Verband der Automobilindustrie (VDA), Berlin

[7] Demirel Y (2012) Energy. Production, conversion, storage, conservation, and coupling. Green energy and technology. Springer, London

[8] Mitschke M, Wallentowitz H (2014) Dynamik der Kraftfahrzeuge, 5. Aufl. Springer Vieweg, Wiesbaden 